\title{
MODULAR AND DISTRIBUTIVE SEMILATTICES
}

\author{
BY
}

JOE B. RHODES

\begin{abstract}
A modular semilattice is a semilattice $S$ in which $w \geqslant$ $a \wedge b$ implies that there exist $x, y \in S$ such that $x \geqslant a, y \geqslant b$ and $x \wedge y=$ $x \wedge w$. This is equivalent to modularity in a lattice and in the semilattice of ideals of the semilattice, and the condition implies the Kurosh-Ore replacement property for irreducible elements in a semilattice. The main results provide extensions of the classical characterizations of modular and distributive lattices by their sublattices: A semilattice $S$ is modular if and only if each pair of elements of $S$ has an upper bound in $S$ and there is no retract of $S$ isomorphic to the nonmodular five lattice. A semilattice is distributive if and only if it is modular and has no retract isomorphic to the nondistributive five lattice.
\end{abstract}

1. Introduction. The first published definition of distributive semilattice was given by Grätzer and Schmidt [7], and this concept was studied further in [8] and [9]. The defining condition for modular semilattices is apparently due to Edmondson [5], who employed this concept in the study of the lattice of ideals of a modular lattice. The first announcement of this as a defining condition for modular semilattices appeared in [11]. At about the same time, a study of modularity in partially ordered groupoids was published by Varlet [14]. An equivalent definition was known to Grätzer and Schmidt, and subsequently appeared in Grätzer's book [6]. It is likely that other workers in the field have invented equivalent definitions and have proved some of the elementary results, although none of this work has been available to the author. R. Balbes [1] has investigated a semilattice generalization of distributive lattices that is not equivalent to the one given here.

2. Definitions and elementary results. A semilattice is a set $S$ with a commutative, associative, idempotent binary operation $\wedge$ on $S$. A partial order may be defined on $S$ by $a \leqslant b$ iff $a \wedge b=a$. A semilattice $S$ is distributive iff $w \geqslant a \wedge b$ implies that there exist $x$ and $y$ in $S$ such that $x \geqslant a, y \geqslant$ $b$ and $x \wedge y=w$. $S$ is modular iff $w \geqslant a \wedge b$ implies that there exist $x$ and

Presented to the Society, November 6,1969 and August 19,1970 under the titles Modular semilattices and $A$ characterization of modular semilattices by their retracts; received by the editors March 31, 1971.

AMS (MOS) subject classifications (1970). Primary 06A20; Secondary 06A30.

Key words and phrases. Lattice, semilattice, poset, modular, distributive, semimodular, ideal, retract, neet-irreducible, finite maximal chain. 
$y$ in $S$ such that $x \geqslant a, y \geqslant b$ and $x \wedge y=x \wedge w$. A semilattice is conditionally complete when every nonempty subset with a lower bound has an infimum. A semilattice is complete when it is conditionally complete and has a zero. A nonempty subset $A$ of a semilattice is called an ideal provided $a \wedge b \in$ $A$ iff $a \in A$ and $b \in A$. A subset $T$ is convex in $S$ iff $x, y \in T, z \in S$ and $x \leqslant z \leqslant y$ implies that $z \in T$. An interval of $S$ is a convex subsemilattice in which each pair of elements has an upper bound. The set $[a, b]=\{x \mid a \leqslant$ $x \leqslant b\}$ is called the closed interval from $a$ to $b$.

There are a number of elementary results which follow readily from these definitions. We shall give only a brief account of these here, leaving the details for the reader. It is easy to prove that the defining conditions for modular and distributive semilattices are equivalent to the usual definitions in a lattice setting and that every distributive semilattice is modular. Each pair of elements of a modular semilattice $S$ has an upper bound in $S$, consequently conditionally complete modular and distributive semilattices are lattices. Unlike the lattice case, subsemilattices do not inherit modularity or distributivity, although convex subsemilattices do if they contain upper bounds for each pair of elements in the subsemilattice.

The ideals of a semilattice $S$, ordered by set inclusion, form a complete joint semilattice $\hat{S}$, which becomes a lattice iff each pair of elements in $S$ has an upper bound in $S$. As in the lattice case, a semilattice is modular (distributive) if and only its lattice of ideals is modular (distributive). A version of Dedekind's transposition principle holds in a modular semilattice, in that if $a \vee b$ exists in $S$, then the map $f:[a, a \vee b] \rightarrow[a \wedge b, b]$ defined by $f(x)=b \wedge x$ is an isomorphism. Since the inverse $f^{-1}(x)=a \vee x$ exists in this case, so does $a \vee x$ for each $x \in[a \wedge b, b]$.

A semilattice $T$ is called a retract of a semilattice iff there are homomorphisms $f: S \rightarrow T$ and $g: T \rightarrow S$ such that $f g$ is the identity on $T$. Clearly, $f$ is an epimorphism and $g$ is a monomorphism under these conditions. If $R$ is a subsemilattice of $S$ and there is an epimorphism $h: S \rightarrow R$ such that $h \mid R$ is the identity, then $R$ is certainly a retract of $S ; h$ in this case is called a retraction.

Although epimorphs and subsemilattices of modular and distributive semilattices do not inherit these respective properties, subsemilattices which are also retracts are better behaved. One can prove

THEOREM 2.1. A retract of a modular (distributive) semilattice is modular (distributive).

This theorem may be used to prove that the product of semilattices is modular (distributive) if and only if each component is modular (distributive). 
The next results are useful in the sequel.

THEOREM 2.2. If $a, b \in S$, then the semilattices $L(b), M(a)$ and $[a, b]$ are retracts of $S$.

Proof. The maps

$$
\begin{aligned}
& f: S \rightarrow L(b) \text { defined by } f(x)=x \wedge b \\
& g: S \rightarrow M(a) \text { defined by } g(x)= \begin{cases}x & \text { if } x \geqslant a ; \\
a & \text { if } x \neq a\end{cases} \\
& h: S \rightarrow[a, b] \text { defined by } h(x)= \begin{cases}x \wedge b & \text { if } x \wedge b \geqslant a ; \\
a & \text { if } x \wedge b \neq a\end{cases}
\end{aligned}
$$

are all epimorphisms.

There are a number of equivalent forms of the definition of modularity ((2) is due to Grätzer and Schmidt):

(1) $w \geqslant a \wedge b$ implies that there exist $x \geqslant a, y \geqslant b$ in $S$ such that $x \wedge y=x \wedge w=y \wedge w$.

(2) $w \geqslant a \wedge b$ and $w \leqslant a$ implies that there exists $y$ in $S$ such that $y \geqslant b$ and $y \wedge a=w$.

(3) $a \wedge b=b \wedge c$ implies that there exists $y$ in $S$ such that $y \geqslant b$ and $y \wedge a=a \wedge c$.

(4) $a \wedge b=b \wedge c$ implies that there exists $y$ in $S$ such that $y \geqslant b$ and $y \wedge a=y \wedge c=a \wedge c$.

Two equivalent forms of distributivity are sometimes useful:

(1) $w \geqslant a \wedge b$ implies that there exists $y$ in $S$ such that $y \geqslant b, y \geqslant w$ and $y \wedge a=a \wedge w$.

(2) $a \wedge b=b \wedge c$ implies that there exists $y$ in $S$ such that $y \geqslant b$, $y \geqslant c$ and $y \wedge a=a \wedge c$.

A semilattice is called semimodular if $b$ covers $a \wedge b$ whenever $a \vee b$ exists and covers $a$. This terminology is due to Dilworth and Crawley [4] and is not equivalent to that given by Birkhoff [2] and Ore [10], even in a lattice. The transposition principle for modular semilattices immediately implies that every modular semilattice is semimodular. By modifying the proof given by Szász [13, p. 104], one may extend the Croisot-Szász lattice results with finite maximal chains to semimodular semilattices. Indeed, this result holds in a poset: Suppose $P$ is a poset such that if $a \vee b$ exists in $P$ and covers $a$, then $a \wedge b$ exists in $P$ and is covered by $b$. Under these conditions, if there is a finite maximal chain from $a$ to $b$ in $P$, then every chain from $a$ to $b$ is finite and all maximal chains from $a$ to $b$ have the same length. It should be noted that the weaker hypotheses used by Birkhoff and Ore are not adequate to support the stronger conclusion of the above theorem. The defining condition for semimodular 
semilattices is called the upper covering condition by Szász. He also defines a lower covering condition and notes that both are true in a modular lattice. It can be easily seen that the lower covering condition may fail in a modular semilattice.

3. Main results. This section deals primarily with the characterization of modular and distributive semilattices by their retracts, and to a lesser extent, the extension of the Kurosh-Ore theorem to semilattices.

We first consider the latter problem. An element $x$ of a semilattice is called (meet) irreducible if $x=a$ or $x=b$ whenever $x=a \wedge b$. A set $M$ of irreducible elements is said to be a (meet) representation of $x$ if $x=\wedge M$; a representation $M$ of $x$ is irredundant if $\Lambda(M \backslash m\})>x$ for all $m \in M$. The relationship between distributivity and meet representations discovered by Birkhoff carries over without modification to the semilattice case: Every element of a distributive semilattice has at most one finite irredundant meet representation by irreducibles. A proof for the lattice case that works equally well for semilattices may be found in Szász [13, p. 99].

The modular case has attracted more attention, with special emphasis on the ability to substitute within irreducible representations when they are not unique. Following Crasley [3], we say that an element $x$ has replaceable irredundant representations if for every pair of irredundant representations $x=\Lambda Q=$ $\Lambda Q^{1}$ and each $q \in Q$, there exists $q^{1} \in Q^{1}$ such that $x=q^{1} \wedge \Lambda(Q \backslash q)$. It is known that $[2, \mathrm{p} .75]$

THEOREM 3.1. Suppose $S$ is a semilattice in which $a \wedge b$ is irreducible in $[a \wedge b, a]$ whenever $b$ is irreducible in $S$. Then irredundant finite representations in $S$ are replaceable. As a corollary, finite irredundant representations of an element have the same number of components.

The following result is the converse of Theorem 3.1.

THEOREM 3.2. Let $S$ be a semilattice in which each element has a finite representation by irreducibles. If all irredundant finite representations are replaceable then $a \wedge b$ is irreducible in $[a \wedge b, a]$ whenever $b$ is irreducible in $S$.

Proof. Let $a=\Lambda M$ be a finite representation of $a$ by irreducibles. If the element $b$ may be omitted from the representation $a \wedge b=\Lambda M \wedge b$, then $a \wedge b=a$ and the conclusion is immediate. Otherwise, there is an irredundant representation $a \wedge b=a_{1} \wedge a_{2} \wedge \cdots \wedge a_{q} \wedge b$ where $\left\{a_{1}, a_{2}, \cdots, a_{q}\right\} \subseteq M$. Suppose now that $a \wedge b=x \wedge y$ with $x, y \leqslant a$. Let $x=\Lambda X$ and $y=\Lambda Y$ be finite irreducible representations of $x$ and $y$, respectively. Since the representations are finite, there is an irredundant representation $a \wedge b=x_{1} \wedge x_{2} \wedge$ 
$\cdots \wedge x_{n}$ where $\left\{x_{1}, x_{2}, \cdots, x_{n}\right\} \subseteq X \cup Y$. By the replacement property, one of these elements, say $x_{i}$, may be substituted for $b$ so that $a_{1} \wedge a_{2} \wedge \cdots$ $\wedge a_{q} \wedge x_{i}=a \wedge b$. Since $x_{i} \in X \cup Y, x_{i} \geqslant x$ or $x_{i} \geqslant y$. In the first case, $x=a \wedge\left(x_{i} \wedge x\right)=\left(a \wedge x_{i}\right) \wedge x=(a \wedge b) \wedge x=a \wedge b$, and in the second case, $y=a \wedge b$. This proves that $a \wedge b$ is irreducible in $[a \wedge b, a]$.

The example given in Szász [13, p. 43] is a nonmodular lattice in which Theorem 3.2 holds.

Results similar to Theorems 3.1 and 3.2 have been obtained for the compactly generated case by Crawley [3].

We now show that Theorem 3.1 applies to modular semilattices.

THEOREM 3.3. Let $S$ be a modular semilattice. If $x_{2}$ is irreducible in $S$, then $x_{1} \wedge x_{2}$ is irreducible in $\left[x_{1} \wedge x_{2}, x_{1}\right]$.

Proof. Suppose $x_{1} \wedge x_{2}=b \wedge c$ with $b, c \leqslant x_{1}$. By the preceding section, there exist $t \geqslant x_{2}, z \geqslant x_{2}$ such that $t \wedge x_{1}=b$ and $z \wedge x_{1}=c$. Thus $t \wedge z \geqslant x_{2}$ and $(z \wedge t) \wedge x_{1}=b \wedge c=x_{1} \wedge x_{2}$. Moreover, there exists $y \geqslant x_{1}$ such that $y \wedge(z \wedge t)=(z \wedge t) \wedge x_{2}=x_{2}$. Since $x_{2}$ is irreducible, $x_{2}=y$ or $x_{2}=z \wedge t$. If $x_{2}=y$, then $x_{1} \wedge x_{2}=x_{1}$ is irreducible in $\left[x_{1} \wedge x_{2}, x_{1}\right]$. Suppose $x_{2}=z \wedge t$. Since $x_{2}$ is irreducible, $x_{2}=z$ or $x_{2}=t$. In the first case, $x_{1} \wedge x_{2}=c$, and in the second $x_{1} \wedge x_{2}=b$. This proves that $x_{1} \wedge x_{2}$ is irreducible in $\left[x_{1} \wedge x_{2}, x_{1}\right]$.

Corollary 3.4 (Kurosh-Ore Theorem for Semilattices). The conclusion of Theorem 3.1 is true in a modular semilattice.

In another work we have investigated the question of the existence of representations in semilattices [12].

We now turn our attention to the main results of this paper. Since modularity and distributivity are not inherited by subsemilattices, it is necessary to turn to retracts for an appropriate generalization of the classical characterizations of modular and distributive lattices by their sublattices.

THEOREM 3.5. The retract relation $R=\{(A, B) \mid A$ and $B$ are semilattices and $B$ is a retract of $A\}$ is reflexive and transitive.

LEMma 3.6. Suppose $S$ is a semilattice, $L$ is a subsemilattice of $S$ and $f(x)=V_{L}\{y \in L \mid y \leqslant x\}$ is defined for each $x \in S$. If $f(x) \leqslant x$ for all $x$ in some subsemilattice $A$ of $S$, then $f \mid A$ is a homomorphism.

Proof. Suppose $x, y \in A$. Since $x \wedge y \leqslant x$,

$$
\begin{aligned}
f(x \wedge y) & =V_{L}\{z \in L \mid z \leqslant x \wedge y\} \\
& \leqslant V_{L}\{z \in L \mid z \leqslant x\}=f(x),
\end{aligned}
$$


and, similarly, $f(x \wedge y) \leqslant f(y)$. Suppose $x, y \in A$ and $t=f(x) \wedge f(y)$. Since $f(x) \leqslant x$ and $f(y) \leqslant y, t \leqslant x \wedge y$. Thus $t \leqslant V_{L}\{z \in L \mid z \leqslant x \wedge y\}=f(x \wedge y)$. This proves $f(x) \wedge f(y)=f(x \wedge y)$.

A subsemilattice $L$ of $S$ is called a sublattice of $S$ when $L$ is a lattice and the insertion map $i: L \rightarrow S$ defined by $i(x)=x$ is a lattice monomorphism. If $L$ is complete, $i\left(V_{L} A\right)=V i(A)$, and $i\left(\Lambda_{L} A\right)=\Lambda_{s} i(A)$ also, $L$ is called a complete sublattice.

THEOREM 3.7. If $L$ is a complete sublattice of a semilattice $S$, then $L$ is a retract of $S$. Thus a finite sublattice of $S$ is a retract of $S$.

Proof. Let $L$ be a complete sublattice of a semilattice $S$ and let $a=$ $\Lambda L, b=V L$. Since $[a, b]$ is a retract of $S$, it suffices to show that $L$ is a retract of $[a, b]$. Define $f:[a, b] \rightarrow L$ by $f(x)=V_{L}\{y \in L \mid y \leqslant x\}$. Since $L$ is complete, $f$ is well defined. Since $L$ is a complete sublattice, $f(x) \leqslant x$ for all $x \in[a, b]$. Thus $f=f \|[a, b]$ is a homomorphism by Lemma 3.6, and since $f$ is surjective, it is an epimorphism.

It was shown by Dedekind that a lattice is modular if and only if it contains no sublattice isomorphic to the nonmodular five lattice. It is easy to show that a modular semilattice may contain a nonmodular five lattice as a subsemilattice. The next two examples show that a nonmodular semilattice may fail to have a sublattice isomorphic to the nonmodular five lattice, even if each pair of elements has an upper bound. (The nonmodular five lattice $N_{5}$ is defined in [2].)

Example 3.8. Let $N_{1}=\{(0,0),(0,1),(0,2),(1,0)\} \cup A$ where $A=$ $\{(1,2+1 / n) \mid n$ is a positive integer $\}$. With the coordinatewise order of the plane, $N_{1}$ is a nonmodular semilattice; there is no sublattice of $N_{1}$ isomorphic to the nonmodular five lattice.

ExAmple 3.9. Let $N_{2}=\{(0,0),(0,1),(1,0)\} \cup A \cup B$ with $A=$ $\{(0,1+1 / n) \mid n$ is a positive integer $\}$ and $B=\{(1,1+1 / n) \mid n$ is a positive integer\}. With the coordinatewise order of the plane, $N_{2}$ is a nonmodular semilattice. There is no sublattice of $N_{2}$ isomorphic to the nonmodular five lattice.

The correct semilattice generalization of Dedekind's theorem is provided by the following result.

THEOREM 3.10. A semilattice $S$ is modular if and only if each pair of elements in $S$ has an upper bound and $S$ does not contain a retract isomorphic to the nonmodular five lattice.

Proof. Necessity. If $S$ is modular, then each pair of elements has an upper bound. If $R$ is a retract of $S$, then $R$ is modular by Theorem 2.1. Therefore $R$ is a modular lattice if $R$ is a lattice, and thus $R$ cannot be isomorphic to the nonmodular five lattice. 
Sufficiency. Suppose $S$ is nonmodular and each pair of elements in $S$ has an upper bound. We shall construct a retract isomorphic to the nonmodular five lattice. Since $S$ is nonmodular, there exist $c \geqslant a \wedge b$ with $c \leqslant a$ such that $y \wedge a \neq c$ for all $y \geqslant b$. Let $t \geqslant a, b$. Then $a=t \wedge a>c$. Since $c \geqslant a \wedge b$, $a \wedge b \leqslant b \wedge c$; and since $a \geqslant c, a \wedge b \geqslant b \wedge c$. Thus, $a \wedge b=b \wedge c$. Let $u$ be an upper bound for $a$ and $b$ and let $L=\{a, b, c, a \wedge b, u\}$. We shall prove that $L$ is a retract of $[a \wedge b, u]$. For this purpose, let $W=\{x \in$ $[a \wedge b, u] \mid x \geqslant b$ and $x \geqslant c\}$ and define for all $x \in[a \wedge b, u]$,

$$
f(x)= \begin{cases}u & \text { if } x \in W, \\ b & \text { if } x \geqslant b \text { and } x \neq c, \\ c & \text { if } x \neq b, x \geqslant c \text { and } x \neq a \wedge z \text { for every } z \in W, \\ a & \text { if } x \neq b \text { and } x \geqslant a \wedge z \text { for some } z \in W, \\ a \wedge b & \text { if } x \neq b \text { and } x \neq c .\end{cases}
$$

We must show $f$ is a homomorphism. Let $x, y \in[a \wedge b, u]$. There are five cases. The interesting case is the first.

Case 1. $f(x)=a$. Then $x \geqslant b$ and $x \geqslant a \wedge z$ for some $z \in W$. Since $x \neq b, x \wedge y \geqslant b$ for all $y \in[a \wedge b, u]$. Suppose $f(y)=a$. Then $y \geqslant a \wedge w$ for some $w \in W$, so $x \wedge y \geqslant a \wedge(z \wedge w)$ and $z \wedge w \in W$. Thus $f(x \wedge y)=$ $a=f(x) \wedge f(y)$. Suppose $f(y)=u$. Then $x \wedge y \geqslant a \wedge(z \wedge y)$ and $z \wedge y \in$ $W$, so $f(x \wedge y)=a=f(x) \wedge f(y)$. Suppose $f(y)=c$. Since $y \neq a \wedge p$ for every $p \in w, x \wedge y \geqslant a \wedge p$ for every $p \in W$. Since $z \in W, x \geqslant a \wedge z \geqslant$ $a \wedge c=c$, so $x \wedge y \geqslant c$. Hence $f(x \wedge y)=c=f(x) \wedge f(y)$. Suppose $f(y)=b$ or $f(y)=a \wedge b$. Then $y \neq c$, so $x \wedge y \neq c$; since $x \wedge y \neq b, f(x \wedge y)=$ $a \wedge b=f(x) \wedge f(y)$.

Case 2. $f(x)=u$. The case $f(y)=a$ has been considered. If $f(y)=b$, $x \wedge y \geqslant b$ and $x \wedge y \neq c$, so $f(x \wedge y)=b=f(x) \wedge f(y)$. If $f(y)=c$, $x \wedge y \geqslant c, x \wedge y \neq b$ and $x \wedge y \neq a \wedge z$ for every $z \in W$. Hence $f(x \wedge y)=$ $c=f(x) \wedge f(y)$. If $f(y)=a \wedge b, x \wedge y \neq b$ and $x \wedge y \neq c$, so $f(x \wedge y)=$ $a \wedge b=f(x) \wedge f(y)$.

The remaining cases $f(x)=a \wedge b, f(x)=c$ and $f(x)=b$ are easily handled. This proves that $L$ is an epimorph of $[a \wedge b, u]$ and since it is obviously a subsemilattice, $L$ is a retract of $[a \wedge b, u]$. By Theorems 2.2 and 3.5, then, $L$ is a retract of $S$.

Notice that Theorem 3.10 contains the classical case because every finite sublattice of a lattice is a semilattice retract of that lattice.

EXAMPLE 3.11. Let $M_{5}=\{(0,0),(0,2),(1,1),(2,0),(2,2)\}$. In the coordinatewise order of the plane $M_{5}$ is a modular, nondistributive lattice. $M_{5}$ is called the nondistributive five lattice [2]. 
EXAMPLE 3.12. Let $A=\{(2, x) \mid 2<x \leqslant 3\} \cup\{(0,0),(0,2),(1,1)$, $(2,0)\}$. In the order of the plane, $A$ is a modular, nondistributive semilattice. No sublattice of $A$ is isomorphic to $M_{5}$.

EXAMPLE 3.13. Let $B=\{(2, y) \mid 1<y \leqslant 2\} \cup\{(0,0),(2,0),(1,1)\} \cup$ $\{(0, y) \mid 1<y \leqslant 2\}$. In the coordinatewise order of the plane $B$ is a modular, nondistributive semilattice which does not contain a sublattice isomorphic to $M_{5}$.

Birkhoff discovered that a lattice is distributive if and only if it is modular and there is no sublattice isomorphic to $M_{5}$. The next theorem is the semilattice generalization of this.

THEOREM 3.14. A semilattice $S$ is distributive if and only if $S$ is modular and there is no retract of $S$ isomorphic to the nondistributive five lattice.

Proof. $(\Rightarrow$ ) Apply Theorem 2.1.

$\Leftrightarrow$ Suppose $S$ is modular and nondistributive. As in Theorem 3.10, we construct a retract isomorphic to the nondistributive five lattice. Since $S$ is not distributive, there exist $b \geqslant d \wedge e$ such that $x \wedge y \neq b$ for all $x \geqslant d$ and $y \geqslant e$. By modularity, there exist $a \geqslant d, c \geqslant e$ such that $a \wedge b=b \wedge c=$ $a \wedge c$. Since $b \geqslant a \wedge c$ we must have $b>a \wedge c$ by the assumption. If $a=$ $a \wedge c$, then $b \geqslant a \geqslant d$; let $t$ be an upper bound for $b$ and $e$, and then $t \wedge b=b$ with $t \geqslant e, b \geqslant d$. Since this is contrary to assumption, $a>a \wedge c$. Similarly $c>a \wedge c$. Moreover, if $x \geqslant a$ and $y \geqslant c, x \wedge y \neq b$.

We show that $x \wedge y \neq a$ for all $x \geqslant b$ and $y \geqslant c$. Suppose $x \wedge y=a$ for some $x \geqslant b$ and $y \geqslant c$. Then $x \wedge c=x \wedge y \wedge c=a \wedge c=b \wedge c$, so by modularity there exists $q \geqslant c$ such that $q \wedge x=x \wedge b=b$. But $q \geqslant c$ and $x \geqslant a$, so this is contrary to assumption. Therefore $x \wedge y \neq a$ for all $x \geqslant b$ and $y \geqslant c$. Similarly, $x \wedge y \neq c$ for all $x \geqslant a$ and $y \geqslant b$.

Let $u$ be an upper bound of $a, b$, and $c$. Let

$$
K=\left\{x \in[a \wedge b, u] \mid x \geqslant a_{1} \wedge a_{2} \wedge a_{3}\right.
$$

for some $a_{1} \geqslant a, b, a_{2} \geqslant a, c$, and $\left.a_{3} \geqslant b, c\right\}$.

Clearly, $K$ is an ideal of $[a \wedge b, u]$.

We first show that $a, b, c, a \wedge b \notin K$. Suppose $a \in K$. Then $a \geqslant a_{1} \wedge$ $\left(a_{2} \wedge a_{3}\right)$ implies that there exist $x \geqslant a_{1}, y \geqslant a_{2} \wedge a_{3}$ such that $x \wedge y=$ $x \wedge a$. But $x \geqslant a_{1} \geqslant a, b$ implies $x \wedge y=a$, and $x \geqslant a_{1} \geqslant b$ and $y \geqslant$ $a_{2} \wedge a_{3} \geqslant c$ contradict an earlier statement. Hence $a \notin K$, and by a similar argument $b, c \notin K$. Since $a \notin K$ and $K$ is an ideal, $a \wedge b \notin K$.

Define a relation $\equiv$ on $[a \wedge b, u]$ as follows: $x \equiv y$ if and only if $x \wedge d=y \wedge d$ for some $d \in K$. It is easily seen that $\equiv$ is a congruence relation. From the above, $a, b, c, a \wedge b \not \equiv$. 
We prove that $a \not \equiv a \wedge b$; the argument that $b \not \equiv a \wedge b, c \not \equiv a \wedge b$ is similar. Suppose $a \wedge b \equiv a$. Then $(a \wedge b) \wedge d=a \wedge d$ for some $d \in K$ implies that $b \geqslant a \wedge d \geqslant a \wedge\left(a_{1} \wedge a_{2} \wedge a_{3}\right)=a \wedge a_{3}$. By modularity, there exist $x \geqslant a, y \geqslant a_{3}$ such that $x \wedge y=y \wedge b=b$ since $y \geqslant a_{3} \geqslant b$. Since $x \geqslant a$ and $y \geqslant a_{3} \geqslant c$, this contradicts an earlier statement. Thus $a \neq a \wedge b$.

We show that $a \not \equiv b$. The proofs that $a \not \equiv c$ and $b \not \equiv c$ are similar. Suppose $a \equiv b$. Then $a \wedge d=d \wedge b$ for some $d \in K$. Then

$$
a \geqslant b \wedge d \geqslant b \wedge a_{1} \wedge a_{2} \wedge a_{3}=b \wedge a_{2}
$$

implies by modularity that there exist $x \geqslant b, y \geqslant a_{2} \geqslant a, c$ such that $x \wedge y=$ $y \wedge a=a$, contrary to an earlier statement.

We now prove that if $x \equiv y, x \geqslant a$, and $y \geqslant b$, then $x \equiv u$. Suppose $x \equiv y, x \geqslant a$ and $y \geqslant b$. There exists $d \in K$ such that $x \wedge d=y \wedge d$. Since $y \geqslant b, x \geqslant x \wedge d=y \wedge d \geqslant b \wedge d \geqslant b \wedge a_{2}$ for some $a_{2} \geqslant a, c$. Since $x \geqslant b \wedge a_{2}$, by modularity there exists $p \geqslant a_{2}, q \geqslant b$ such that $p \wedge q=$ $p \wedge x=q \wedge x$. Since $p \geqslant a_{2} \geqslant a$ and $x \geqslant a, a \wedge q=a \wedge p \wedge q=a \wedge p \wedge$ $x=a$, hence $q \geqslant a$. Since $q \geqslant b, q \in K$. Thus $x \geqslant p \wedge q$, so $x \in K$ and $x \equiv u$. Similarly, it can be shown that if $x \equiv y$, and either $x \geqslant a$ and $y \geqslant c$, or $x \geqslant b$ and $y \geqslant c$, then $x \equiv u$.

Let $N=\{a \wedge b, a, b, c, u\}$. Define $f:[a \wedge b, u] \rightarrow N$, by

$$
f(x)= \begin{cases}u & \text { if } x \in K, \\ a & \text { if } x \notin K \text { and } x \geqslant a \wedge d \text { for some } d \geqslant b, c, \\ b & \text { if } x \notin K \text { and } x \geqslant b \wedge d \text { for some } d \geqslant a, c, \\ c & \text { if } x \notin K \text { and } x \geqslant c \wedge d \text { for some } d \geqslant a, b, \\ a \wedge b & \text { otherwise. }\end{cases}
$$

We first observe that $f$ is well defined. It is impossible that $f(x)=a$ and $f(x)=b$. For suppose $x \notin K, x \geqslant a \wedge d$ for $d \geqslant b, c$ and $x \geqslant b \wedge e$ for $e \geqslant a, c$. Since $x \geqslant a \wedge d$, by modularity there exist $m \geqslant a, n \geqslant d$ such that $m \wedge n=n \wedge x$. Since $n \in K, m \equiv x$. Similarly, there exists $p \geqslant b$ such that $p \equiv x$. Thus $m \equiv p, m \geqslant a$ and $p \geqslant b$, so $x \equiv m \equiv u$ implies $x \in K$, contrary to assumption. Similarly, it is impossible that $f(x)=a$ and $f(x)=c$, or that $f(x)=b$ and $f(x)=c$.

It has been shown that $a, b, c \notin K$; hence it is easily seen that $f(a)=a$, $f(b)=b$, and $f(c)=c$. Obviously, $f(u)=u$. Notice also that if $a \wedge b \geqslant a \wedge d$ for some $d \geqslant b, c$, then $(a \wedge b) \wedge d=a \wedge d$. Hence $a \wedge b \equiv a$, contrary to a previous result. This shows that $f(a \wedge b) \neq a$, and, similarly, $f(a \wedge b) \neq b$, $f(a \wedge b) \neq c$. Since $a \wedge b \notin K, f(a \wedge b)=a \wedge b$.

It is left to show that $f$ is a homomorphism. Suppose $x, y \in[a \wedge b, u]$. 
First of all, it is obvious that the sets $f^{-1}(u), f^{-1}(a \wedge b), f^{-1}(a), f^{-1}(b)$ and $f^{-1}(c)$ are closed under meet. The remaining cases are considered below.

Case I. $f(x)=a \wedge b$. Suppose $f(y)=a$. Then $y \notin K$ and $y \geqslant a \wedge d$ for some $d \geqslant b, c$. Since $K$ is an ideal, $x \wedge y \notin K$. If $x \wedge y \geqslant a \wedge e$ for $e \geqslant b, c$, then $f(x)=a$. Hence $f(x \wedge y) \neq a$, and similarly, $f(x \wedge y) \neq b$ and $f(x \wedge y) \neq c$. Thus $f(x \wedge y)=a \wedge b$. A similar argument shows that when $f(y)=b$ or $f(y)=c, f(x \wedge y)=a \wedge b=f(x) \wedge f(y)$. Suppose $f(y)=u$. Then $y \in K$ but $x \wedge y \notin K$ because $x \notin K$. Moreover, if $f(x \wedge y)=a, b$, or $c$, then $f(x)=a, b$ or $c$ respectively. Thus $f(x \wedge y)=a \wedge b=f(x) \wedge f(y)$.

Case II. $f(x)=u$ is trivial.

Case III. $f(x)=a$ (the cases $f(x)=b$ and $f(x)=c$ are similar to this). In view of preceding remarks it suffices to consider the case $f(y)=b$ (the case $f(y)=c$ is analogous). Thus $x, y \notin K, x \geqslant a \wedge d$ for $d \geqslant b, c$ and $y \geqslant$ $b \wedge e$ for $e \geqslant a, c$. Clearly $x \wedge y \notin K$ because $K$ is an ideal. If $f(x \wedge y)=$ $a, b$ or $c$, then $f(x)=f(y)=a, b$ or $c$ respectively. Thus $f(x \wedge y)=a \wedge b=$ $f(x) \wedge f(y)$.

This proves that $N=\{a \wedge b, a, b, c, u\}$ is a retract of $[a \wedge b, u]$. Since $[a \wedge b, u]$ is a retract of $S$ by Theorem 2.2, it follows from Theorem 3.5 that $N$ is a retract of $S$. It is clear from the construction of $N$ that $N$ is isomorphic to the nondistributive five lattice, and this completes the proof.

I am grateful to Professor Don Edmondson for his advice during the preparation of this paper and to the National Science Foundation for partial support through a Summer Traineeship at the University of Texas in 1969.

\section{BIBLIOGRAPHY}

1. R. Balbes, $A$ representation theory for prime and implicative semilattices, Trans. Amer. Math. Soc. 136 (1969), 261-267. MR 38 \#2062.

2. G. Birkhoff, Lattice theory, 3rd ed., Amer. Math. Soc. Colloq. Publ., vol. 25, Amer.. Math. Soc., Providence, R. I., 1967. MR 37 \#2638.

3. P. Crawley, Decomposition theory for nonsemimodular lattices, Trans. Amer. Math. Soc. 99 (1961), 246-254. MR 22 \#10930.

4. R. P. Dilworth and P. Crawley, Decomposition theory for lattices without chain conditions, Trans. Amer. Math. Soc. 96 (1960), 1-22. MR 22 \#9461.

5. D. E. Edmondson, Homomorphisms of a modular lattice, Unpublished Doctoral Dissertation, California Institute of Technology, Pasadena, California, 1954.

6. G. Grätzer, Lattice theory, Freeman, San Francisco, Calif., 1971.

7. G. Grätzer and E. T. Schmidt, On congruence lattices of lattices, Acta Math. Acad. Sci. Hungar. 13 (1962), 179-185. MR 25 \#2983.

8. C. Green, A decomposition property for semilattices, Notices Amer. Math. Soc. 15 (1968), 1040. Abstract \#68T-A55.

9. T. Katriňák, Pseudokomplementäre Halbverbände, Mat. Časopis Sloven. Akad. Vied. 18 (1968), 121-143. MR 41 \#6733. 
10. O. Ore, Chains in partially ordered sets, Bull. Amer. Math. Soc. 49 (1943), 558566. MR 5, 88 .

11. J. B. Rhodes, Modular semilattices, Notices Amer. Math. Soc. 17 (1970), 272273. Abstract \#672-659.

12. Decomposition of semilattices with applications to topological lattices, Pacific J. Math. 44 (1973), 299-307. MR 47 \#3262.

13. G. Szász, Introduction to lattice theory, 3rd rev. ed., Akad. Kiadó, Budapest; Academic Press, New York, 1963. MR 29 \#3396.

14. J. C. Varlet, Modularity and distributivity in partially ordered groupoids, Bull. Soc. Roy. Sci. Liège 38 (1969), 639-648. MR 43 \#3189.

MERCK \& COMPANY, INC., RAHWAY, NEW JERSEY 07065 